\title{
Limits of Input-to-State Stability
}

\author{
Fritz Colonius \\ Institut FÜr Mathematik, Universität Augsburg \\ 86135 Augsburg/Germany, Colonius@math.uni-Augsburg.De \\ WOLFGANG KLIEMANN \\ Department of Mathematics \\ Iowa State University \\ Ames IowA 50011
}

September 11, 2002

\section{IntRODUCTION ${ }^{1}$}

The fundamental notion of input-to-state-stability relates the admissible inputs and initial values to the maximal amplitudes of the corresponding trajectories (Sontag [7].) For a smooth control system on $\mathbb{R}^{n}$

$$
\dot{x}(t)=f(x(t), u(t))
$$

with inputs $u$ taking values in a set $U \subset \mathbb{R}^{m}$ containing zero, we use the following local version of Input-to-State-Stability (compare, e.g., Grüne [5]).

Definition 1. A positively invariant compact set $A$ is called Input-to-State-Stable (ISS) for $\rho_{1}>0$ on a neighborhood $B$ of $A$ with attraction rate $\beta$ of class $\mathcal{K} \mathcal{L}$ and robustness gain $\gamma$ of class $\mathcal{K}_{\infty}$ if the following inequality holds for every $x \in B$ and every input $u$ with $\|u\|_{\infty}<\rho_{1}$ :

$$
\|\varphi(t, x, u)\|_{A} \leq \max \left\{\beta\left(\|x\|_{A}, t\right), \gamma\left(\|u\|_{\infty}\right)\right\} \text { for all } t \geq 0 .
$$

Here $\|\cdot\|_{A}$ denotes the distance to the set $A$. Since $\gamma(0)=0$, it follows that $A$ is attracting for the unperturbed system with $u=0$; and near $A$ there are attracting sets for small perturbation ranges. The supremal $\rho_{1}$-value with this property determines the maximal robustness of the attractor $A$ and its properties will be discussed in this paper. We will restrict our attention to invariant sets $A$ with the ISS-property.

The ingredients of this definition are attracting sets and their behavior under perturbations with varying maximal amplitudes. In order to get more insight into the behavior of systems under perturbations and the ISS-property, we will start with an

\footnotetext{
${ }^{1} \mathrm{~A}$ preliminary version of this paper has appeared as [3].
} 
attractor for the unperturbed system; then we discuss the behavior under perturbations with $\|u\|_{\infty} \leq \rho$, for varying $\rho>0$. In particular we will relate the loss of the attractivity property at $\rho_{1}$ to a change in the controllability behavior; see Corollary 16.

There are a huge variety of different notions for attractors in control theory and dynamical systems theory. We will use notions going back to the work of C. Conley that are well established in dynamical systems theory. They will also be used for the perturbed system via the notion of control flows.

We consider the following class of systems

$$
\dot{x}(t)=f(x(t), u(t)), u \in \mathcal{U},
$$

where $f$ is $C^{1}$ and $\mathcal{U}=\left\{u \in L_{\infty}\left(\mathbb{R}, \mathbb{R}^{m}\right), u(t) \in U\right.$ for almost all $\left.t \in \mathbb{R}\right\}$. We assume that unique global solutions $\varphi(t, x, u)$ exist for $t \in \mathbb{R}$. We also assume that the vector space $V$ spanned by these smooth vector fields,

$$
V=\operatorname{span}\{f(\cdot, u), u \in U\}
$$

is finite dimensional and that the set of admissible vector fields

$$
F=\{f(\cdot, u), u \in U\}
$$

is compact and convex. The time-dependent vector fields taking values in $F$ are

$$
\mathcal{F}=\left\{v \in L_{\infty}(\mathbb{R}, V), v(t) \in F \text { for } t \in \mathbb{R}\right\}
$$

System (2) defines a continuous flow on $\mathcal{F} \times \mathbb{R}^{n}($ with weak*-topology on $\mathcal{F})$

$$
\Phi_{t}(v, x)=(v(t+\cdot), \varphi(t, x, v)), t \in \mathbb{R}
$$

here $v(t+\cdot)(s)=v(t+s), s \in \mathbb{R}$, denotes the shifted vector field. We call this the associated non-parametric control flow. It is closely related to control flows as considered in [1] with the shift on the space $\mathcal{U}$ of control functions; here the time dependent vector fields are parametrized by the control functions and it has to be assumed that the system is control-affine and the control range $U$ is compact and convex. Nonparametric control flows inherit all properties of control flows, mainly due to the fact that the shift on $\mathcal{F}$ is chain transitive. Details will appear elsewhere.

For simplicity we suppose that everything is contained in the interior of a compact invariant and connected set $K=\mathrm{cl} \operatorname{int} K \subset \mathbb{R}^{n}$. Thus we consider the control flow on the compact metric space $\mathcal{F} \times K$. Furthermore we will need some technical condition assuring that the system moves away fast enough from the boundary of $K$; see (12), 
(13). Throughout we require local accessibility, i.e., the reachable sets in positive and negative time,

$$
\begin{aligned}
& \mathcal{O}_{\leq T}^{+}(x)=\{\varphi(t, x, u), 0 \leq t \leq T \text { and } u \in \mathcal{U}\} \\
& \mathcal{O}_{\leq T}^{-}(x)=\{\varphi(-t, x, u), 0 \leq t \leq T \text { and } u \in \mathcal{U}\}
\end{aligned}
$$

have for all $x \in K$ and all $T>0$ nonvoid interiors. This is satisfied under the usual accessibility rank condition.

Remark 2. The apparently very restrictive assumption on existence of a compact invariant set $K$ can often be achieved if the involved vector fields are smoothly changed outside a large ball. Then one has to add in an appropriate way invariant sets "near infinity". The technical details are somewhat involved and hence will not be presented here.

\section{Attractors and Chain Control Sets}

First we discuss the behavior on the level of chain control sets or, equivalently, of chain transitivity for the control flow. In the next section we combine this with control sets.

By a flow on a compact metric space $X$ with metric denoted by d we mean a continuous map $\Phi: \mathbb{R} \times X \rightarrow X$ with

$$
\Phi(0, x)=x \text { and } \Phi(t, \Phi(s, x))=\Phi(t+s, x) \text { for all } s, t \in \mathbb{R} \text { and } x \in X \text {. }
$$

Complete proofs of the results stated here are given, e.g., in [1], Appendix B; see Robinson [6] for the relation to gradient-like systems and Lyapunov functions.

Definition 3. For a flow $\Phi$ on a compact metric space $X$ a compact invariant set $A$ is an attractor if it admits a neighborhood $N$ such that $A=\omega(N)=\{y \in X$, there are $t_{k} \rightarrow \infty$ and $x_{k} \in N$ with $\left.\Phi\left(t_{k}, y_{k}\right) \rightarrow y\right\}$.

We also allow the empty set as an attractor. A neighborhood $N$ as in Definition 3 is called an attractor neighborhood. Every attractor is compact and invariant, and a repeller is an attractor for the time reversed flow (with limit sets denoted by $\left.\omega^{*}(N)\right)$. Every attractor comes with an associated complementary repeller $A^{*}=\{x \in X, \omega(x) \cap A\}=\varnothing$. Then for every $x \notin A \cup A^{*}$

$$
\omega^{*}(x) \subset A^{*} \text { and } \omega(x) \subset A \text {. }
$$

For all considered flows we assume that there are only finitely many connected components of the chain recurrent set $\mathcal{R}$, which consists of all $x \in X$ such that for all $\varepsilon, T>0$ there is an $(\varepsilon, T)$-chain from $x$ to $x$ which is given by $k \in \mathbb{N}$, points 
$x_{0}=x, x_{1}, \ldots, x_{k}=x$ in $X$ and times $T_{i}>T$ with $\mathrm{d}\left(\Phi\left(T_{i}, x_{i}\right), x_{i+1}\right)<\varepsilon$ for $i=0, \ldots, k-1$. The connected components of the chain recurrent set (called the chain recurrent components) are chain transitive, i.e., they consist of points which can be connected by $(\varepsilon, T)$-chains for all $\varepsilon, T>0$. It turns out that an attractor $A$ consists of chain recurrent components together with the connecting trajectories. To be more precise define for chain recurrent components $\mathcal{E}, \mathcal{E}^{\prime}$

$$
\left[\mathcal{E}, \mathcal{E}^{\prime}\right]=\left\{x \in X, \omega^{*}(x) \subset \mathcal{E} \text { and } \omega(x) \subset \mathcal{E}^{\prime}\right\}
$$

Then attractors are of the following form.

Theorem 4. Let $\Phi$ be a flow on a compact metric space $X$ with the finitely many chain recurrent components $\mathcal{E}_{1}, \ldots, \mathcal{E}_{l}$. Then for every attractor $A$ there is an index set $J=J(A) \subset\{1, \ldots, l\}$ such that

$$
A=\bigcup_{i, j \in J}\left[\mathcal{E}_{i}, \mathcal{E}_{j}\right]
$$

A Morse decomposition consists of finitely many subsets $\left\{\mathcal{M}_{1}, \ldots, \mathcal{M}_{k}\right\}$ of $X$ (called Morse sets) such that there is a strictly increasing sequence of attractors

$$
\emptyset=A_{0} \subset A_{1} \subset A_{2} \subset \ldots \subset A_{k}=X
$$

with

$$
\mathcal{M}_{k-i}=A_{i+1} \cap A_{i}^{*}, 0 \leq i \leq k-1 .
$$

A finest Morse decomposition exists if and only if the chain recurrent set has finitely many connected components; its Morse sets are these chain recurrent components.

We denote the chain recurrent components of the unperturbed system $\dot{x}=f(x, 0)$ in $K$ by $E_{1}^{0}, \ldots, E_{l}^{0}$, and consider a compact set $A^{0} \subset K$ which is an attractor. By Theorem 4 there is an index set $J=J\left(A^{0}\right)$ such that

$$
A^{0}=\bigcup_{i, j \in J}\left[E_{i}^{0}, E_{j}^{0}\right] .
$$

For a description of the chain recurrent components of the control system, we recall that a chain control set $E$ is a maximal subset of the state space $K$ such that for all $x \in E$ there is $u \in \mathcal{U}$ with $\varphi(t, x, u) \in E$ for all $t \in \mathbb{R}$ and for every two elements $x, y$ and all $\varepsilon, T>0$ there are $k \in \mathbb{N}$ and $x_{0}=x, x_{1}, \ldots, x_{k}=y$ in $K, u_{0}, \ldots, u_{k} \in \mathcal{U}$ and $T_{0}, \ldots, T_{k-1}>T$ with $\mathrm{d}\left(\varphi\left(T_{i}, x_{i}, u_{i}\right), x_{i+1}\right)<\varepsilon$. Their meaning for the control flow is indicated in the following proposition (cp. [1, Theorem 4.1.4]), where $f(\cdot, u)$ denotes the vector field-valued function

$$
t \mapsto f(\cdot, u(t)) \in \mathcal{F} \subset L_{\infty}(\mathbb{R}, V) .
$$


Proposition 5. Let $E \subset K$ be a chain control set of system (2). Then

$$
\mathcal{E}=\left\{(f(\cdot, u), x) \in \mathcal{F} \times \mathbb{R}^{n}, u \in \mathcal{U} \text { and } \varphi(t, x, u) \in E \text { for all } t \in \mathbb{R}\right\}
$$

is a chain recurrent component for the control flow $(\mathcal{F} \times K, \Phi)$. Conversely, let $\mathcal{E} \subset \mathcal{F} \times K$ be a chain recurrent component for the control flow. Then the projection of $\mathcal{E}$ to $K$ is a chain control set.

In order to allow for different maximal amplitudes of the inputs, we consider the ranges $U^{\rho}=\rho \cdot U, \rho \geq 0$. It is easily seen that the corresponding trajectories coincide with the trajectories $\varphi^{\rho}(t, x, u)$ of

$$
\dot{x}(t)=f^{\rho}(x(t), u(t))=f(x(t), \rho u(t)), u \in \mathcal{U} .
$$

Obviously, the chain recurrent components $E_{i}^{0}$ of the unperturbed system are contained in chain control sets $E_{i}^{\rho}$ of the $\rho$-system for every $\rho>0$. For positive $\rho$ every chain recurrent component of the flow for the $\rho$-system is the lift $\mathcal{E}_{i}^{\rho}$ of a chain control set. Sadly, for (arbitrarily small) positive $\rho$-values, there may exist chain control sets $E^{\rho}$ not containing a chain recurrent component of the unperturbed system. An easy example is obtained by looking at systems where for some $\rho_{0}>0$ a saddle node bifurcation occurs in $\dot{x}=f(x, \rho)$. A more intricate example is [1], Example 4.7.8. We will ignore this fact here, mainly, because we cannot contribute much to its analysis. Instead we concentrate on the chain recurrent components $\mathcal{E}_{i}^{\rho}, i=1, \ldots, l$, corresponding to the chain recurrent components of the unperturbed system. Observe that for increasing $\rho$-values they may intersect and hence coincide and change attraction properties; it is this process that we will analyze. Next we state the situation for the attractors.

Proposition 6. Assume that for every $\rho>0$ every chain recurrent component contains a chain recurrent component $E_{i}^{0}$ of the unperturbed system. Then there is $\rho_{0}>0$ such that for all $\rho$ with $\rho_{0}>\rho>0$ the attractors $\mathcal{A}^{\rho}$ of the $\rho$-system are given by

$$
\mathcal{A}^{\rho}=\bigcup_{i, j \in J}\left[\mathcal{E}_{j}^{\rho}, \mathcal{E}_{k}^{\rho}\right]
$$

where the allowed index sets $J$ coincide with those for $\varrho=0$. The chain recurrent components $\mathcal{E}_{i}^{\rho}$ depend upper semicontinuously on $\rho$ and converge for $\rho \rightarrow 0$ toward $\mathcal{F} \times E_{i}^{0} ;$ all $\mathcal{E}_{j}^{\rho}$ are different.

Proof. Every attractor for $\rho$ is a union of chain transitive components and the corresponding intervals. Since chain transitive components converge for $\rho \rightarrow 0$ towards those of the system with $\rho=0([1$, Corollary 3.4.8]) this also follows for the intervals. Furthermore note that $\mathcal{E}_{j}^{\rho} \subset \mathcal{E}_{j}^{\rho^{\prime}}$ for $\rho^{\prime}>\rho \geq 0$. Hence the chain transitive 
components $\mathcal{E}_{j}^{\rho}$ contained in $\mathcal{A}^{\rho}$ must satisfy $j \in J$ and for $\rho$ small enough they are different.

Looking at the notion of Input-to-State Stability, we observe that in this context only those attractors are of interest which are input-global in the following sense: The attraction property should hold for arbitrary inputs $u \in \mathcal{U}^{\rho}$. This is not part of the definition of attractors. Luckily, this is automatically satisfied for control flows as shown by the following proposition (essentially, this is due to chain transitivity of $\mathcal{F}$.)

Proposition 7. Consider an attractor $\mathcal{A}$ for the nonparametric control flow $\Phi$ associated to system (2). Then it has an attractor neighborhood of the form $\mathcal{F} \times B$ with $B \subset K$, i.e., $\mathcal{A}=\omega(\mathcal{F} \times B)$.

Proof. If $\mathcal{A}^{*}$ is the complementary repeller for $\mathcal{A}$, then the distance between the projections $A$ and $A^{*}$ to $\mathbb{R}^{n}$ of $\mathcal{A}$ and $\mathcal{A}^{*}$, respectively, is greater than some positive number $\delta$. In fact: Otherwise, by compactness, there are $x \in A \cap A^{*}$ and $u, u^{*} \in \mathcal{U}$ with $(f(\cdot, u), x) \in A$ and $\left(f\left(\cdot, u^{*}\right), x\right) \in \mathcal{A}^{*}$. Define $w \in \mathcal{U}$ by

$$
w(t)=\left\{\begin{array}{cc}
u^{*}(t) & \text { for } t \leq 0 \\
u(t) & \text { for } t>0
\end{array} .\right.
$$

By definition of the topology on $\mathcal{F}$, one finds $\omega(f(\cdot, w), x)=\omega(f(\cdot, u), x)$ and $\omega^{*}(w, x)=$ $\omega^{*}\left(u^{*}, x\right)$. Hence invariance of $\mathcal{A}$ and $\mathcal{A}^{*}$ implies $(f(\cdot, w), x) \in \mathcal{A}$ and $\omega^{*}(f(\cdot, w), x) \subset$ $\mathcal{A}^{*}$. This contradicts $\mathcal{A} \cap \mathcal{A}^{*}=\varnothing$.

Now let $v \in \mathcal{F}$ be arbitrary and take $x$ in the $\delta / 2$-neighborhood $B$ of $A$. If $\omega(v, x)$ is not contained in $\mathcal{A}$, then $(v, x)$ is in the complementary repeller $\mathcal{A}^{*}$. But then the distance between $A^{*}$ and $A$ must be smaller than $\delta / 2$. This contradiction shows that $(v, x)$ is in an attractor neighborhood of $\mathcal{A}$.

Thus we obtain the following characterization of sets $I \subset K$ which are the projections of attractors.

Proposition 8. $A$ set $A \subset K$ is the projection of an attractor $\mathcal{A} \subset \mathcal{F} \times K$ if and only if it has a neighborhood $B \subset K$ such that $A$ equals

$$
\left\{x \in K, \text { there are } u_{n} \in \mathcal{U}, x_{n} \in B \text { and } t_{n} \rightarrow \infty \text { with } \varphi\left(t_{n}, x_{n}, u_{n}\right) \rightarrow x\right\} .
$$

Proof. Let $\mathcal{A}$ be an attractor with projection $A$. By Proposition 7 every attractor neighborhood is of the form $\mathcal{F} \times B$ with a neighborhood $B$ of the projection $A$ to $K$. Hence (4) follows. Conversely, suppose that $A \subset K$ has a neighborhood $B$ with (4). Then the set

$$
\mathcal{A}=\mathcal{F} \times A .
$$


is compact invariant and has the attractor neighborhood $\mathcal{F} \times B$.

The discussion up to now completely describes the attractor properties for small positive $\rho$. Here the attractors and the chain control sets reflect the properties of the unperturbed system. For larger $\rho$-values this need not be the case, because chain control sets contained in the attractor $\mathcal{A}^{\rho}$ may merge with other chain control sets. We cannot describe the changes in the attractor properties on the level of chain control sets only. Instead we have to go to control sets, which is possible under additional assumptions.

\section{Loss of Attractivity and Control Sets}

The purpose of this section is to describe loss of the attraction property when the input range is increased. According to Proposition 6, attractors are sets of the form

$$
\mathcal{A}^{\rho}=\bigcup_{i, j \in J}\left[\mathcal{E}_{i}^{\rho}, \mathcal{E}_{j}^{\rho}\right] .
$$

Recall that a control set $D$ is a maximal controlled invariant set (in $K$ ) such that

$$
D \subset \operatorname{clO}^{+}(x) \text { for all } x \in D \text {; }
$$

here $\mathcal{O}^{+}(x)=\{\varphi(t, x, u), t>0$ and $u \in \mathcal{U}\}$ denotes the reachable set from $x$. A control set is an invariant control set if equality holds in (6). For simplicity we assume that all control sets are in the interior of $K$. By local accessibility, all invariant control sets have nonvoid interiors.

We will assume that for all $\rho$ with $\rho_{1}>\rho>0$ the chain control sets $E_{i}^{\rho}$ are the closures of control sets $D_{i}^{\rho}$ with nonvoid interior; observe that some of the control sets in the attractor must be invariant, since every point can be steered into an invariant control set. We also lift control sets $D^{\rho}$ via

$$
\mathcal{D}^{\rho}=\operatorname{cl}\left\{(f(\cdot, u), x) \in \mathcal{F} \times \mathbb{R}^{n}, u \in \mathcal{U}^{\rho} \text { and } \varphi(t, x, u) \in \operatorname{int} D \text { for all } t \in \mathbb{R}\right\}
$$

Then $E_{i}^{\rho}=\operatorname{cl} D_{i}^{\rho}$ implies $\mathcal{E}_{i}^{\rho}=\mathcal{D}_{i}^{\rho}$. It follows that the attractors are given by

$$
\mathcal{A}^{\rho}=\bigcup_{i, j \in J}\left[\mathcal{D}_{i}^{\rho}, \mathcal{D}_{j}^{\rho}\right] \text {. }
$$

We will analyze the case where for $\rho=\rho_{1}$ the set $\mathcal{A}^{\rho_{1}}$ has lost the attractor property. The following example illustrates the main issues involved.

Example 9. Consider a locally accessible system contained in a compact set $K \subset \mathbb{R}^{n}$ with five control sets

$$
C_{1}, C_{2}, D_{1}, D_{2}, D_{3}
$$


where $C_{1}$ and $C_{2}$ are invariant control sets, $D_{1}$ is open, and

$$
\begin{aligned}
& D_{2} \subset \mathcal{O}^{+}\left(D_{1}\right), C_{1} \subset \mathcal{O}^{+}\left(D_{2}\right), \\
& D_{3} \subset \mathcal{O}^{+}\left(D_{2}\right), C_{2} \subset \mathcal{O}^{+}\left(D_{3}\right) .
\end{aligned}
$$

and it is not possible to steer the system from $\mathrm{cl}_{3}$ to $C_{1}$, i.e., $\mathcal{O}^{+}(x) \cap C_{1}=\varnothing$ for all $x \in \operatorname{cl} D_{3}$ :

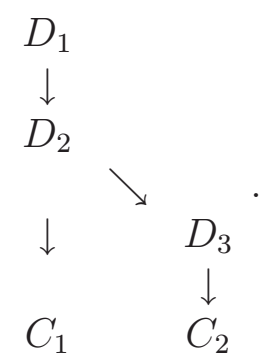

Assume furthermore that the closures of these control sets are the chain control sets. Then there is the following increasing sequence of attractors:

$$
\begin{aligned}
& \mathcal{A}_{0}=\varnothing, \mathcal{A}_{1}=\mathcal{C}_{1}, \mathcal{A}_{2}=\mathcal{C}_{1} \cup \mathcal{C}_{2}, \\
& \mathcal{A}_{3}=\mathcal{A}_{2} \cup \mathcal{D}_{3} \cup\left[\mathcal{D}_{3}, \mathcal{C}_{2}\right] \\
& \mathcal{A}_{4}=\mathcal{A}_{3} \cup\left[\mathcal{D}_{2}, \mathcal{C}_{1}\right] \cup\left[\mathcal{D}_{2}, \mathcal{C}_{2}\right] \cup\left[\mathcal{D}_{2}, \mathcal{D}_{3}\right], \\
& \mathcal{A}_{5}=\mathcal{A}_{4} \cup \mathcal{D}_{1} \cup\left[\mathcal{D}_{1}, \mathcal{D}_{2}\right] \cup\left[\mathcal{D}_{1}, \mathcal{D}_{3}\right] \cup\left[\mathcal{D}_{1}, \mathcal{C}_{1}\right] \cup\left[\mathcal{D}_{1}, \mathcal{C}_{2}\right]=\mathcal{F} \times K,
\end{aligned}
$$

with corresponding repellers

$$
\begin{aligned}
& \mathcal{A}_{0}^{*}=\mathcal{F} \times K, \\
& \mathcal{A}_{1}^{*}=\mathcal{D}_{1} \cup \mathcal{D}_{2} \cup \mathcal{D}_{3} \cup \mathcal{C}_{2} \cup\left[\mathcal{D}_{1}, \mathcal{D}_{2}\right] \cup\left[\mathcal{D}_{1}, \mathcal{D}_{3}\right] \cup\left[\mathcal{D}_{1}, \mathcal{C}_{2}\right] \cup\left[\mathcal{D}_{2}, \mathcal{D}_{3}\right] \cup\left[\mathcal{D}_{2}, \mathcal{C}_{2}\right] \cup\left[\mathcal{D}_{3}, \mathcal{C}_{2}\right], \\
& \mathcal{A}_{2}^{*}=\mathcal{D}_{1} \cup \mathcal{D}_{2} \cup \mathcal{D}_{3} \cup\left[\mathcal{D}_{1}, \mathcal{D}_{2}\right] \cup\left[\mathcal{D}_{1}, \mathcal{D}_{3}\right] \cup\left[\mathcal{D}_{2}, \mathcal{D}_{3}\right], \\
& \mathcal{A}_{3}^{*}=\mathcal{D}_{1} \cup \mathcal{D}_{2} \cup\left[\mathcal{D}_{1}, \mathcal{D}_{2}\right], \\
& \mathcal{A}_{4}^{*}=\mathcal{D}_{1} \\
& \mathcal{A}_{5}^{*}=\varnothing .
\end{aligned}
$$

This sequence yields the finest Morse decomposition

$$
\mathcal{M}_{5-i}=\mathcal{A}_{i+1} \cap \mathcal{A}_{i}^{*}, i=0,1, \ldots, 4,
$$

which consists of the lifted (chain) control sets:

$$
\begin{aligned}
& \mathcal{M}_{5}=\mathcal{A}_{1} \cap \mathcal{A}_{0}^{*}=\mathcal{C}_{1}, \\
& \mathcal{M}_{4}=\mathcal{A}_{2} \cap \mathcal{A}_{1}^{*}=\mathcal{C}_{2}, \\
& \mathcal{M}_{3}=\mathcal{A}_{3} \cap \mathcal{A}_{2}^{*}=\mathcal{D}_{3}, \\
& \mathcal{M}_{2}=\mathcal{A}_{4} \cap \mathcal{A}_{3}^{*}=\mathcal{D}_{2}, \\
& \mathcal{M}_{1}=\mathcal{A}_{5} \cap \mathcal{A}_{4}^{*}=\mathcal{D}_{1} .
\end{aligned}
$$


Observe that one can obtain this finest Morse decomposition also by other increasing attractor sequences, e.g.,

$$
\begin{aligned}
& \mathcal{A}_{0}=\varnothing, \mathcal{A}_{1}=\mathcal{C}_{2}, \\
& \mathcal{A}_{2}=\mathcal{C}_{2} \cup \mathcal{D}_{3} \cup\left[\mathcal{D}_{3}, \mathcal{C}_{2}\right] \\
& \mathcal{A}_{3}=\mathcal{A}_{2} \cup \mathcal{C}_{1} \\
& \mathcal{A}_{4}=\mathcal{A}_{3} \cup\left[\mathcal{D}_{2}, \mathcal{C}_{1}\right] \cup\left[\mathcal{D}_{2}, \mathcal{C}_{2}\right] \cup\left[\mathcal{D}_{2}, \mathcal{D}_{3}\right] \\
& \mathcal{A}_{5}=\mathcal{A}_{4} \cup \mathcal{D}_{1} \cup\left[\mathcal{D}_{1}, \mathcal{D}_{2}\right] \cup\left[\mathcal{D}_{1}, \mathcal{D}_{3}\right] \cup\left[\mathcal{D}_{1}, \mathcal{C}_{1}\right] \cup\left[\mathcal{D}_{1}, \mathcal{C}_{2}\right]=\mathcal{U} \times K
\end{aligned}
$$

Now consider what may happen when the input range is increased with $\rho$ : The corresponding (chain) control sets increase. If $C_{2}$ intersects the closure of $D_{3}$, there is no essential change in the attractor structure: attractors containing the lift of $C_{2}$ also contain the lift of $D_{3}$. Things are different, if $C_{1}$ and the closure of $D_{2}$ intersect. Then the attractor $\mathcal{C}_{1}$ has vanished. It can only be recovered as part of an attractor containing also $\mathcal{C}_{2}$.

The situation alluded to at the end of the previous example is characterized by the facts that the invariant control set $C_{1}$ is strictly contained in a chain control set; and arbitrarily close to $C_{1}$ there are points which can be steered into another invariant control set. Then one finds the control set $D_{2}$ such that its lift is contained in every attractor containing $\mathcal{C}_{1}$. Thus the loss of the attraction property of the attractor $\mathcal{C}_{1}$ is accompanied by this phenomenon. In order to analyze it, we introduce the following notation.

Definition 10. For a set $I \subset K$ the domain of attraction is

$$
\mathbf{A}(I)=\left\{x \in K, \operatorname{clO}^{+}(x) \cap \operatorname{int} I \neq \varnothing\right\},
$$

and the invariant domain of attraction is

$$
\mathbf{A}^{i n v}(I)=\left\{x \in K, \begin{array}{c}
\text { if } C \subset \operatorname{clO}^{+}(x) \text { is an invariant } \\
\text { control set, then } C \subset I
\end{array}\right\} .
$$

We note the following simple result.

Proposition 11. If $I \subset \operatorname{int} \mathbf{A}(I)$, then the domain of attraction $\mathbf{A}(I)$ is open in $K$. The invariant domain of attraction $\mathbf{A}^{i n v}(I)$ is closed in $K$.

Proof. Openness of $\mathbf{A}(I)$ follows from continuous dependence on initial values. For closedness of $\mathbf{A}^{i n v}(I)$ consider $x_{j} \in \mathbf{A}^{i n v}(I)$ with $x_{j} \rightarrow x$. Suppose that $\operatorname{clO}^{+}(x) \cap$ $C^{\prime} \neq \varnothing$ for an invariant control set $C^{\prime}$. This implies $\mathcal{O}^{+}(x) \cap \operatorname{int} C^{\prime} \neq \varnothing$ and hence 
there are $u \in \mathcal{U}$ and $T>0$ with $\varphi(T, x, u) \in \operatorname{int} C^{\prime}$. Hence for $j$ large enough also $\varphi\left(T, x_{j}, u\right) \in \operatorname{int} C^{\prime}$ implying $C^{\prime} \subset I$ and hence $x \in \mathbf{A}^{i n v}(I)$.

We will analyze the case where $I$ is the projection to $\mathbb{R}^{n}$ of an invariant set $\mathcal{I} \subset \mathcal{F} \times \mathbb{R}^{n}$ of the form

$$
\mathcal{I}=\bigcup_{i, j \in J(I)}\left[\mathcal{D}_{i}, \mathcal{D}_{j}\right]
$$

for the lifts of control sets $D_{j}$ assuming that the invariant domain of attraction $\mathbf{A}^{i n v}(I)$ is nonvoid. Then at least one of the involved control sets is invariant and every point in $I$ can be steered in the interior of one of the invariant control sets contained in $I$. Hence, by continuous dependence on initial values, it follows that the projection $I$ of $\mathcal{I}$ to $\mathbb{R}^{n}$ is contained in the interior of its domain of attraction $\mathbf{A}(I)$. We will later consider the special case where $\mathcal{I}$ is not an attractor due to the fact that the projection $I$ to $\mathbb{R}^{n}$ intersects the boundary of its invariant domain of attraction,

$$
I \cap \partial \mathbf{A}^{i n v}(I) \neq \varnothing .
$$

Thus arbitrarily close to $I$ one finds points $x$ such that for some control $u \in \mathcal{U}$ one can steer the system away from $I$ into an invariant control set (and then stay there.) Hence it is clear that in this case $\mathcal{I}$ is not an attractor. If for increasing input range the attractors are strictly increasing, it will also follow that they must contain other invariant control sets. We will show that (10) implies that the set $I$ merges with some variant control set as discussed in the example above.

The analysis below will be based on constructing control sets in the open set

$$
L=\bigcup_{j \in J^{\text {out }}}\left[\mathbf{A}(I) \cap \mathbf{A}\left(C_{j}\right)\right] \cap \operatorname{int} K, .
$$

where the index $J^{\text {out }}$ denotes all invariant control set $C_{j}$ with $C_{j} \cap I=\varnothing$. We assume that $L$ is nonvoid. A control set $D \subset L$ with nonvoid interior is called an $L$-invariant control set if $x \in D$ and $\varphi(t, x, u) \notin D$ for some $t>0$ and $u \in \mathcal{U}$ implies $\varphi(t, x, u) \notin L$.

We impose the following strong invariance conditions describing the behavior near the boundary of $K$ :

$$
\begin{array}{r}
\text { For all } x \in L \text { there is } \varepsilon_{x}>0 \text { with } \\
\mathrm{d}(\varphi(t, x, u), \partial K) \geq \varepsilon_{x} \text { for } u \in \mathcal{U} \text { and } t \geq 0 .
\end{array}
$$

There is $\varepsilon_{0}>0$ such that for all $x \in \operatorname{cl} L$ and $u \in \mathcal{U}$

$$
y=\lim _{k \rightarrow \infty} \varphi\left(t_{k}, x, u\right) \in L \text { for } t_{k} \rightarrow \infty \text { implies } \mathrm{d}(y, \partial K) \geq \varepsilon_{0}
$$

We need some topological properties of the boundary of $L$. Let $\partial L$ and $\partial^{K} L$ denote the boundaries of $L$ in $\mathbb{R}^{n}$ and $K$, respectively, and define

$$
\partial_{I} L=\partial^{K} L \cap \mathbf{A}(I), \partial_{j} L=\partial^{K} L \cap \mathbf{A}\left(C_{j}\right) \text { for } j \in J^{\text {out }}, \partial_{0} L=\partial L \cap \partial K .
$$


Note that for each $y \in \partial^{K} L$ the following alternative holds: if $y \in \mathbf{A}(I)$ then $y \notin$ $\mathbf{A}\left(C_{j}\right)$ for every $j \in J^{\text {out }}$; or $y \notin \mathbf{A}(I)$ and there is (at least one) $j \in J^{\text {out }}$ with $y \in \mathbf{A}\left(C_{j}\right)$; this follows since the domains of attraction are open. Abbreviate

$$
\partial_{\text {out }} L=\bigcup_{j \in J^{\text {out }}} \partial_{j} L .
$$

Then

$$
\partial^{K} L=\partial_{I} L \cup \partial_{\text {out }} L \text { and } \partial L=\partial_{I} L \cup \partial_{\text {out }} L \cup \partial_{0} L .
$$

Using again that the domains of attraction are open, one sees that in $\partial^{K} L$ the sets $\partial_{I} L$ and $\partial_{\text {out }} L$ are open and their closures are disjoint. In the topology of $\mathbb{R}^{n}$

$$
y \in \operatorname{cl} \partial_{I} L \cap \mathrm{cl}\left[\bigcup_{j \in J^{\text {out }}} \partial_{j} L\right] \quad \text { implies } y \in \partial_{0} L \subset \partial K .
$$

Note further that $\partial^{K} L$ contains at least one $\partial_{j} L, j \in J^{\text {out }}$. Analogous definitions can be given for every connected component of $L$ and all properties stated above remain valid.

The following technical lemma is needed.

Lemma 12. For every $x \in L$ there are $J_{x}^{\text {out }} \subset J^{\text {out }}$ and $y \in \mathcal{O}^{+}(x)$ such that $y \in$ $\mathbf{A}(I) \cap \bigcap_{j \in J_{x}^{\text {out }}} \mathbf{A}\left(C_{j}\right)$ and $J_{x}^{\text {out }}$ is a minimal index set in the following sense:

If $\varphi(t, y, u) \in L$ for some $t>0$ and $u \in \mathcal{U}$, then $\varphi(t, y, u) \in \mathbf{A}(I) \cap \bigcap_{j \in J_{x}^{\text {out }}} \mathbf{A}\left(C_{j}\right)$.

Proof. Since $x \in L$, there is an index set $J_{1} \subset J^{\text {out }}$ with $x \in \mathbf{A}(I) \cap$ $\bigcap_{j \in J_{1}} \mathbf{A}\left(C_{j}\right)$. If there are $t_{1}>0$ and $v_{1} \in \mathcal{U}$ with $y_{1}:=\varphi\left(t_{1}, x, v_{1}\right) \in L \backslash \bigcap_{j \in J_{1}} \mathbf{A}\left(C_{j}\right)$, then there exists a proper nonvoid subset $J_{2} \subset J_{1}$ with $y_{1} \in \mathbf{A}(I) \cap \bigcap_{j \in J_{2}} \mathbf{A}\left(C_{j}\right)$. Proceeding recursively, one ends up, after finitely many steps, at a point $y \in \mathcal{O}^{+}(x)$ with a minimal index set $J_{x}^{\text {out }}$.

Note that a minimal index set has at least one element. Furthermore, using exact controllability in the interior of control sets, the lemma implies that for each $L$ invariant control set $D$ there is an index set $J_{x}^{\text {out }} \subset J^{\text {out }}$ such that for each $x \in$ int $D$ the index set $J_{x}^{\text {out }}$ is minimal. We cite the following result [1, Proposition 3.3.7] (slightly specialized for our purpose).

Proposition 13. Let $L$ be an open subset of $K$ satisfying the no-return condition

$$
\text { if } z \in \operatorname{clO}^{+}(x) \text { for some } x \in L \text { and } \mathcal{O}^{+}(z) \cap L \neq \emptyset \text {, then } z \in L \text {. }
$$

Consider $x \in L$ and assume that there exists a compact set $Q \subset L$ such that for all $y \in \operatorname{cl} \mathcal{O}^{+}(x) \cap L$,

$$
\operatorname{cl} \mathcal{O}^{+}(y) \cap Q \neq \emptyset .
$$

Then there exists an $L$-invariant control set $D \subset \mathrm{clO}^{+}(x)$ and every $L$-invariant control set $D \subset \operatorname{cl} \mathcal{O}^{+}(x)$ satisfies $\operatorname{cl} D \cap Q \neq \emptyset$. 
This will be used in the next proposition to establish existence of an $L$-invariant control set for the set $L$ defined in (11).

Proposition 14. Assume that $K \subset M$ with $K=\operatorname{clint} K$ is a compact connected invariant set for the control system (2) satisfying the strong positive invariance conditions (12) and (13). Consider an invariant set $\mathcal{I}$ of the form (9) and let $x \in \mathbf{A}(I) \cap \bigcap_{j \in J_{x}^{\text {out }}} \mathbf{A}\left(C_{j}\right)$, where $J_{x}^{\text {out }}$ is some minimal index set for $x$. Then there exists an $L$-invariant control set $D \subset \mathrm{clO}^{+}(x)$ with

$$
\partial D \cap \partial_{j} L \neq \emptyset \text { for all } j \in J_{x}^{o u t} \text {. }
$$

Proof. Let $x \in L$ and define

$$
L_{x}=L \cap \operatorname{clO}^{+}(x)
$$

The proof is based on Proposition 13 applied to $L_{x}$ instead of $L$. Condition (15) is obvious by definition of $L_{x}$. In order to construct a compact set $Q$ with (16), we first introduce some notations. Denote the connected component of $L$ that contains $x$ by $L_{x}$, and let

$$
Q_{0}:=\left\{y \in L_{x}, d\left(y, \operatorname{cl} \partial_{0} L_{x}\right) \geq \varepsilon_{x}\right\},
$$

where $\varepsilon_{x}$ is chosen according to the strong invariance condition (12). For $\varepsilon>0$ and a minimal index set $J_{x}^{\text {out }}$ define

$$
\begin{aligned}
& N_{I}(\varepsilon):=\left\{y \in L_{x}, d\left(y, \operatorname{cl} \partial_{I} L_{x}\right) \leq \varepsilon\right\}, \\
& N_{j}(\varepsilon):=\left\{y \in L_{x}, d\left(y, \operatorname{cl} \partial_{\text {out }} L_{x}\right) \leq \varepsilon\right\} \text { for } j \in J_{x}^{\text {out }} ; \\
& Q_{I}(\varepsilon):=\left\{y \in Q_{0}, d\left(y, \operatorname{cl} \partial_{I} L_{x}\right)=\varepsilon\right\}, \\
& Q_{j}(\varepsilon):=\left\{y \in Q_{0}, d\left(y, \operatorname{cl} \partial_{\text {out }} L_{x}\right)=\varepsilon\right\} \text { for } j \in J_{x}^{\text {out }} .
\end{aligned}
$$

Using (14) we can choose $\varepsilon>0$ small enough such that

$$
d\left(\partial_{I} L_{x} \cap Q_{0}, \partial_{\text {out }} L_{x} \cap Q_{0}\right) \geq 5 \varepsilon
$$

Hence the sets $Q_{I}(\varepsilon)$ and $Q_{\text {out }}(\varepsilon)=\bigcup_{j \in J_{x}^{\text {out }}} Q_{j}(\varepsilon)$ are nonvoid, compact, and disjoint with distance at least $3 \varepsilon$. Decreasing, if necessary, the number $\varepsilon$ further, we may assume that $x \in L_{x} \backslash\left[N_{I}(2 \varepsilon) \cup N_{\text {out }}(2 \varepsilon)\right]$. Every trajectory $\{\varphi(t, y, u), t \geq 0\}$ with $y \in \operatorname{clO}^{+}(x) \cap\left(L_{x} \backslash N_{I}(2 \varepsilon)\right)$ that approaches $I$ for $t \rightarrow \infty$ must exit through $\partial_{I} L_{x} \cap$ $Q_{0}$ and must cross $Q_{I}(\varepsilon)$. Every trajectory $\{\varphi(t, y, u), t \geq 0\}$ with $y \in \operatorname{cl} \mathcal{O}^{+}(x) \cap$ $\left(L_{x} \backslash N_{\text {out }}(2 \varepsilon)\right)$ that approaches $C_{j}, j \in J_{x}^{\text {out }}$ for $t \rightarrow \infty$ must exit through $\partial_{j} L_{x} \cap Q_{0}$ 
and must cross $Q_{j}(\varepsilon)$. Furthermore we find that all $\varepsilon>0$ small enough the compact set

$$
\emptyset \neq Q(x, \varepsilon):=\operatorname{clO}^{+}(x) \cap\left[Q_{I}(\varepsilon) \cup Q_{\text {out }}(\varepsilon)\right]
$$

is contained in $L_{x}$. Hence by Proposition 13 the existence of an $L_{x}$-invariant control set $D \subset \operatorname{clO}^{+}(x)$ follows. Obviously, an $L_{x}$-invariant control set is also $L$-invariant. Finally, condition (17) holds, because in the construction above $\varepsilon$ can be made arbitrarily small.

We use this result in order to analyze invariant sets of the form (7) without the attraction property. For the convenience of the reader we also collect our assumptions.

Theorem 15. Consider the control system (2) in $\mathbb{R}^{n}$ and assume that $K \subset \mathbb{R}^{n}$ is a compact invariant and connected set with $K=\mathrm{cl} \operatorname{int} K$. Assume that the strong invariance conditions (12) and (13) are satisfied. Consider an invariant set $\mathcal{I} \subset \mathcal{F} \times K$ of the form

$$
\mathcal{I}=\bigcup_{i, j \in J}\left[\mathcal{D}_{i}, \mathcal{D}_{j}\right]
$$

for lifts of control sets $D_{j}$ and assume that its projection $I$ to $\mathbb{R}^{n}$ intersects the boundary of its invariant domain of attraction, $I \cap \partial \mathbf{A}^{i n v}(I) \neq \emptyset$. Then there exists a variant control set $D$ with nonvoid interior such that

$$
D \subset \mathbf{A}(I) \cap \mathbf{A}(C)
$$

for some invariant control set $C$ with $C \cap I=\varnothing$ and

$$
D \cap I=\varnothing, \operatorname{cl} \mathbf{A}(D) \cap I \neq \varnothing
$$

Proof. By our assumption there exists a point $x \in I \cap \partial \mathbf{A}^{i n v}(I)$. Then $I$ is contained in the interior of its domain of attraction and there are $x_{k} \in \mathbf{A}(I) \backslash \mathbf{A}^{i n v}(I)$ with $x_{k} \rightarrow x$. Thus there are invariant control sets contained in $\mathrm{clO}^{+}\left(x_{k}\right)$ having void intersection with $I$. Since the number of invariant control sets is finite, we may assume that there is a single invariant control set, say $C$, with $C \cap I=\varnothing$ and $C \subset \operatorname{clO}^{+}\left(x_{k}\right)$ for all $k$. Hence $x_{k}$ is in the set $L$ defined in (11). By the preceding proposition we find $u_{k} \in \mathcal{U}$ and $t_{k}>0$ such that $\varphi\left(t_{k}, x_{k}, u_{k}\right) \in \operatorname{int} D_{k}$ for some $L$-invariant control set $D_{k}$. Since the number of $L$-invariant control sets is finite ([1, Theorem 3.3.18]) we may assume that there is a control set $D \subset L$ with these properties for all $k$. One can steer the system from every point of $D$ into $I$ and into $C$. Hence $D \cap I=\varnothing$ and the other properties hold by construction.

As a corollary, we obtain a result showing that loss of attractivity of an invariant set is connected with the 'merger' with a variant control set. It is the main result of this paper. 
Corollary 16. Consider the control system (2) in $\mathbb{R}^{n}$ and assume that $K=\operatorname{cl}$ int $K \subset$ $\mathbb{R}^{n}$ is a compact and connected set which is invariant for the system with input range given by $\rho_{1}>0$. Assume that the strong invariance conditions (12) and (13) are satisfied. Consider the invariant sets in $\mathcal{F}^{\rho} \times K$

$$
\mathcal{I}^{\rho}=\bigcup_{i, j \in J}\left[\mathcal{D}_{i}^{\rho}, \mathcal{D}_{j}^{\rho}\right]
$$

and assume that they are attractors for $\rho<\rho_{1}$ and that the projection $I^{\rho_{1}}$ to $\mathbb{R}^{n}$ of $\mathcal{I}^{\rho_{1}}$ intersects the boundary of its invariant domain of attraction defined in (8), i.e.,

$$
I^{\rho_{1}} \cap \partial \mathbf{A}^{i n v}\left(I^{\rho_{1}}\right) \neq \varnothing .
$$

Then every attractor containing $\mathcal{I}^{\rho_{1}}$ contains a lifted variant control set $\mathcal{D}^{\rho_{1}}$ with $D^{\rho_{1}} \cap I^{\rho_{1}}=\varnothing$.

Proof. By the previous theorem, there is a control set $D^{\rho_{1}}$ for the $\rho_{1}$-system such that $D^{\rho_{1}} \cap I^{\rho_{1}}=\varnothing$ and $\operatorname{cl} \mathbf{A}\left(D^{\rho_{1}}\right) \cap I^{\rho_{1}} \neq \varnothing$. Now consider an attractor neighborhood of an attractor $\mathcal{A}^{\rho_{1}}$ containing $\mathcal{I}^{\rho_{1}}$. By Proposition 7 we may assume that this neighborhood has the form $\mathcal{F}^{\rho_{1}} \times B$. Hence it contains a pair $(u, x)$ with $\omega(u, x) \subset \mathcal{D}^{\rho_{1}}$. Since $\mathcal{D}^{\rho_{1}}$ is contained in a lifted chain control set $\mathcal{E}^{\rho_{1}}$, this implies that $\mathcal{D}^{\rho_{1}}$ is contained in the attractor $\mathcal{A}^{\rho_{1}}$.

Remark 17. Similar constructions have been given in [2] in order to describe the loss of invariance for control sets.

Remark 18. If a chain control set is the closure of a control set, then it depends continuously on $\rho$. This equality holds for all up to at most countably many $\rho$-values under the following inner-pair condition: For all $\rho^{\prime}>\rho \geq 0$ and $(u, x) \in \mathcal{U}^{\rho_{1}} \times K$ there is $T>0$ with $\varphi^{\rho}(T, x, u) \in \operatorname{int} \mathcal{O}^{\rho++}(x)$, or in other words, $\mathcal{O}^{\rho}(x) \subset \operatorname{int} \mathcal{O}^{\rho^{\prime}}(x)$ (see [1], Theorem 4.7.5.) This condition is easily verified for small $\rho>0$ if a Lie-algebra rank condition is satisfied guaranteeing that the trajectories of the uncontrolled system are in the interior of the reachable sets for $\rho>0$ (more information is given in [1], Chapter 4.) For general $\rho>0$ the inner pair condition holds, e.g., for coupled oscillators if the number of perturbations is equal to the degrees of freedom; for a precise statement and more general results see Gayer [4].)

\section{Conclusions}

We obtain the following picture for the relation between input-to-state stability, attractors and control sets: Suppose that $A^{0}$ is a set which has the local ISS property for $\rho_{1}$ and suppose that $\rho_{1}$ is maximal with this property. Then $A^{0}$ is an attractor for the uncontrolled system, hence it is of the form

$$
A^{0}=\bigcup_{i, j \in J\left(A^{0}\right)}\left[E_{i}^{0}, E_{j}^{0}\right]
$$


where $E_{i}^{0}, i \in\{1, \ldots, l\}$ are the attractors of the uncontrolled system and $I\left(A^{0}\right) \subset$ $\{1, \ldots, l\}$ is some index set. Then for small positive $\rho$ one obtains an attractor $\mathcal{A}^{\rho}$ of the $\rho$-system having the form

$$
\mathcal{A}^{\rho}=\bigcup_{i \in J\left(A^{0}\right)}\left[\mathcal{E}_{i}^{\rho}, \mathcal{E}_{j}^{\rho}\right]
$$

here $\mathcal{E}_{i}^{\rho}$ are the lifts of the chain control sets forming around the chain recurrent components $E_{i}^{0}$. Note that the index set has not changed. For the projection $A^{\rho}$ of $\mathcal{A}^{\rho}$ to $K$ and $\mathcal{A}^{0}=\mathcal{F} \times A^{0}$ the Hausdorff-distances

$$
\mathrm{d}_{H}\left(E_{i}^{\rho}, E_{i}^{0}\right), \mathrm{d}_{H}\left(\mathcal{A}_{i}^{\rho}, \mathcal{A}_{i}^{0}\right), \mathrm{d}_{H}\left(A_{i}^{\rho}, A_{i}^{0}\right)
$$

all tend to zero as $\rho \rightarrow 0$. If for all $\rho \in\left(0, \rho_{1}\right)$ the chain control sets are the closures of control sets $D_{i}^{\rho}$ with nonvoid interior, then the maps

$$
\rho \mapsto E_{i}^{\rho} \text { and } \rho \mapsto A^{\rho}
$$

are continuous with respect to the Hausdorff metric. Now consider $\rho=\rho_{1}$ : If the loss of the ISS property is due to the fact that $A^{\rho_{1}}$ intersects the boundary of its invariant domain of attraction, then the index set $J\left(A^{0}\right)$ does no more indicate an attractor, since one can, arbitrarily close to $A^{\rho_{1}}$, find points which can be steered into an invariant control set $C$ which is not contained in $A^{\rho_{1}}$. According to Corollary 16 this is accompanied by the fact that at $\rho=\rho_{1}$ every attractor containing the set $\mathcal{A}^{\rho_{1}}$ also contains the lift of a variant control set $D^{\rho_{1}}$ from which one can steer the system into $C$.

While these observations do not give quantitative information, a conclusion is that variant control sets (similarly to hyperbolic fixed points for ordinary differential equations) play a decisive role in that they limit the robustness properties of attractors and hence the robustness in ISS.

\section{REFERENCES}

[1] F. Colonius and W. Kliemann, The Dynamics of Control, Birkhäuser, 2000.

[2] —_, An invariance radius for nonlinear systems, in Advances in Mathematical Systems. A Volume in Honor of D. Hinrichsen., F. Colonius, U. Helmke, D. Prätzel-Wolters, and F. Wirth, eds., Birkhäuser, 2000, pp. 77-91.

[3] — Input-to-state-stability, attractors and control sets, in Proceedings of the Conference on Mathematical Theory of Networks and Systems (MTNS), University of Notre Dame, Southbend, Indiana (August 12-16 2002), D. Gilliam and J. Rosenthal, eds., 2002. 
[4] T. GAYER, Dissertation, 2002. Universität Augsburg; in preparation.

[5] L. GRüne, Asymptotic Behavior of Dynamical and Control Systems under Perturbation and Discretization, Springer-Verlag, 2002.

[6] C. Robinson, Dynamical Systems. Stability, Symbolic Dynamics, and Chaos, CRC Press Inc., 1995.

[7] E. Sontag, Smooth stabilization implies coprime factorization, IEEE Trans. Aut. Control, 34 (1989), pp. 435-443. 